\title{
Id-I stimulates cell proliferation through activation of EGFR in ovarian cancer cells
}

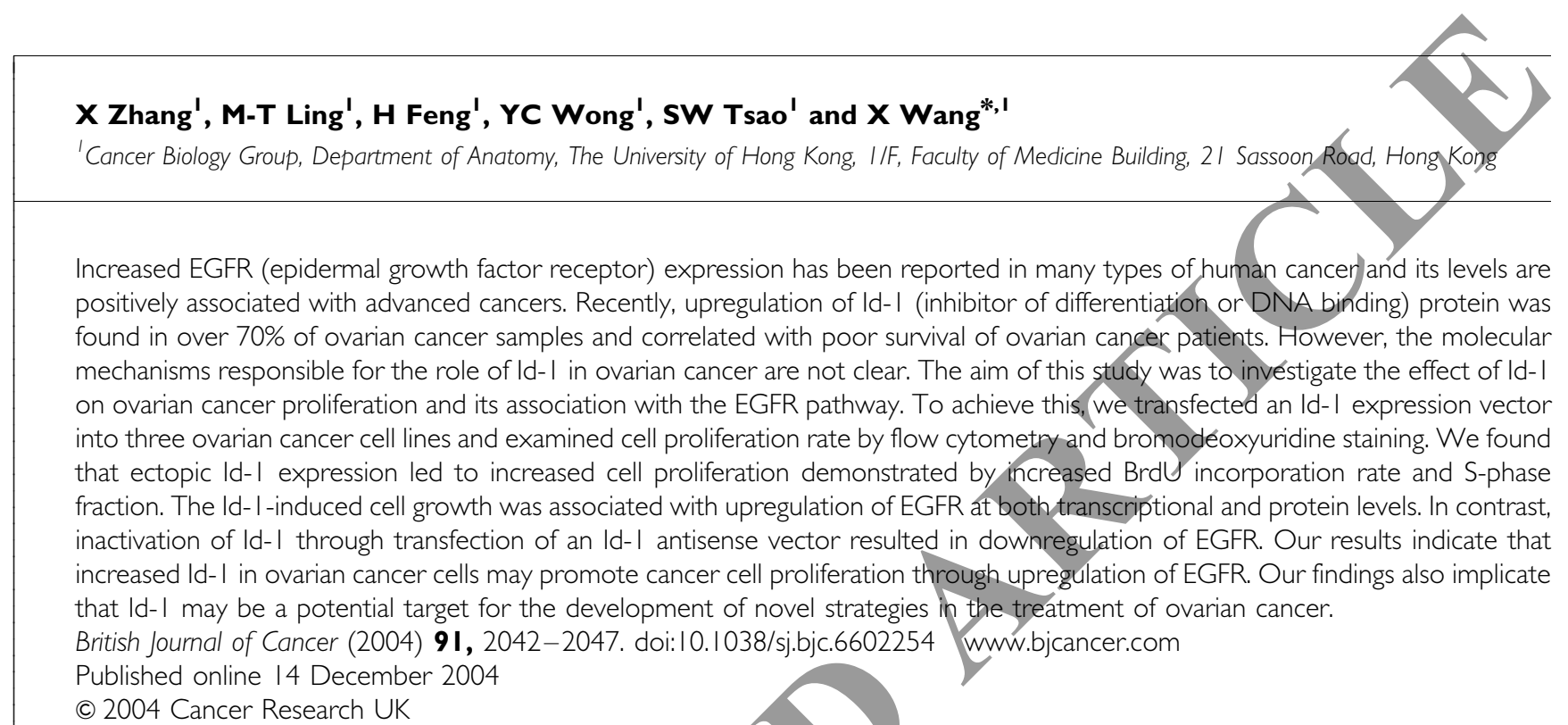

(c) 2004 Cancer Research UK

Keywords: Id-I; EGFR; cell proliferation; ovarian cancer

Ovarian cancer is the second leading cause of death from gynaecologic malignancy worldwide, with a mortality rate of 114.2 per 100000 women (Parkin et al, 2001). Approximately 75\% of women present with ovarian cancer at advanced stage; therefore, prognosis for this disease is poor, with a 5-year survival rate of less than $40 \%$ (Jemal et al, 2004).

Although the molecular basis for the development of ovarian cancer is not clear, upregulation of the epidermal growth factor receptor (EGFR) is reported to be a frequent event in ovarian cancer and associated with tumour progression, invasion and poor survival rate (Kohler et al, 1989; Simpson et al, 1995; Skirnisdottir et al, 2001; Cloven et al, 2004). For example, expression of EGFR is significantly higher in malignant ovarian cancer cells compared to borderline and benign tumours (Simpson et al, 1995). A study on 226 patients with different stages of ovarian cancer found that EGFR-positive staining was evident in approximately $50 \%$ of the patients, and among them $59.8 \%$ had recurrent or died of this disease (Skirnisdottir et al, 2004). Furthermore, in a separate study, the survival time of patients $(n=111)$ with EGFR-positive tumour was much shorter than patients with EGFR-negative tumours (Kohler et al, 1989). These results indicate that EGFR may be a key factor in promoting ovarian cancer growth as well as progression. Recently, using antisense technology, suppression of EGFR led to inhibition of cellular proliferation, cell adhesion and tumorigenicity in ovarian cancer cells (Alper et al, 2000). In

*Correspondence: Dr X Wang; E-mail: xhwang@hkucc.hku.hk Received I 5 July 2004; revised 12 October 2004; accepted 18 October 2004; published online 14 December 2004 addition, targeting the EGFR using an active EGFR-specific tyrosine kinase inhibitor ZD1839 also resulted in the reduction of ovarian cancer cell growth (Sewell et al, 2002). These results further suggest that inactivation of EGFR pathway may provide a therapeutic target for the treatment of ovarian cancer. However, the molecular basis of the EGFR-induced ovarian cancer cell proliferation is still not clear.

Recently, Id-1 (inhibitor of differentiation or DNA binding) has been suggested as one of the upstream regulators of the EGFR pathway (Ling et al, 2004). Id-1 is a member of the helix-loophelix (HLH) transcription factor family. It lacks the basic domain for DNA binding and acts as a dominant inhibitor of the basic HLH transcription factors by forming heterodimers (Benezra et al, 1990). Like EGFR, upregulation of Id-1 is frequently found in many types of human cancer such as breast (Lin et al, 2000), pancreas (Maruyama et al, 1999), cervical (Schindl et al, 2001), head and neck (Langlands et al, 2000) and prostate cancer (Ouyang et al, 2002a), and increased Id-1 expression levels are associated with advanced tumour stage and poor prognosis (Maruyama et al, 1999; Schindl et al, 2001; Ouyang et al, 2002a). Recently, it is reported that over $70 \%$ of ovarian cancer samples (among a total of 101 cases) are found to express Id-1 protein, while none of the nonmalignant cystadenomas shows positive Id-1 staining examined by immunohistochemistry as well as Western blotting (Schindl et al, 2003). In addition, the cancer samples with poor or moderate histological differentiation show stronger Id-1 expression than the well-differentiated tumours. Furthermore, the overall survival is much shorter in the patients with higher Id-1 expression than the patient with relatively lower Id-1 expression (Schindl et al, 2003). These results indicate that Id-1 may play an 
important part not only in tumorigenesis but also progression of ovarian cancer.

The fact that both Id-1 and EGFR protein expression levels increase with progression and poor prognosis as well as chemoresistance of ovarian cancer (Alper et al, 2001; Maihle et al, 2002; Schindl et al, 2003; Cloven et al, 2004) raises a hypothesis that these two proteins may either share similar functions or regulate through same pathways. In addition, evidence from our previous studies has shown that Id-1 promotes prostate cancer cell proliferation through activation of EGFR (Ling et al, 2004), indicating that Id-1 may be an upstream regulator of EGFR. To study the role of Id-1 on ovarian cancer cell growth and its association with EGFR pathway, in this study, we first transfected an Id-1 expression vector into three ovarian cancer cell lines and then examined the effect of ectopic Id-1 expression on ovarian cancer cell proliferation using bromodeoxyuridine (BrdU) staining and flow cytometric analysis. The effect of Id-1 on EGFR expression at both transcriptional and protein levels was also determined by luciferase assay and Western blotting. These results were further verified through transfection of an antisense Id-1 vector in two ovarian cancer cell lines with high levels of Id-1. Our results suggest that ectopic Id-1 expression stimulates ovarian cancer cell proliferation and this process is mediated through upregulation of EGFR. Our results provide novel evidence to suggest Id-1 as an upstream regulator of the EGFR pathway in promoting ovarian cancer cell growth.

\section{MATERIALS AND METHODS}

\section{Cell lines and cell culture conditions}

Five ovarian cancer cell lines, Skov3 (obtained from ATCC, Manassas, VA, USA), Ovca420, Ovca432, Ovca433, Ovca429, were maintained in RPMI 1640 (Life Technologies Inc., Carlsbad, CA, USA) supplemented with $2 \mathrm{~mm}$ L-glutamine and $5 \%\left(\mathrm{vv}^{-1}\right)$ foetal calf serum (FCS) at $37^{\circ} \mathrm{C}$. Ovca420, Ovca432, Ovca433 and Ovca429 were established from freshly isolated ascites or tumour explants from patients with late-stage ovarian adenocarcinomas with distinct characteristics (Rauh-Adelmann et al, 2000).

\section{Generation of stable Id-1-expressing transfectants}

The pBabe-Id-1 retroviral expression vector and its corresponding vector control were used for generation of stable transfectants. Details on the vectors as well as transfection procedures have been described previously (Ouyang et al, 2002b). All the transfectants were selected and maintained in puromycin $\left(1-2 \mu \mathrm{g} \mathrm{ml}^{-1}\right)$. The pool of more than 20 individual clones transfected with either Id-1 or pBabe was generated. Cell culture medium was changed to serum-free medium (SFM) before performing additional experiments.

\section{BrdU staining}

Detailed experimental procedures have been described previously (Wang et al, 2002a). Briefly, monolayer cells were grown on 4-mm Chamber slides (ICN, Biomedicals, Aurora, OH, USA) and the culture medium was changed to SFM for $48 \mathrm{~h}$. Then, the cells were treated with BrdU (10 mM) for $1 \mathrm{~h}$ and then washed once with PBS. The cells were then fixed in cold methanol for $5 \mathrm{~min}$ at room temperature and washed in PBS. The cells were incubated with mouse monoclonal antibody against BrdU (1:10, Roche Diagnostics, Indianapolis, IN, USA) for $1 \mathrm{~h}$ at $37^{\circ} \mathrm{C}$ and then with antimouse IgG-FITC for $1 \mathrm{~h}$ at $37^{\circ} \mathrm{C}$ after washing with PBS. The percentage of FITC-positive cells was evaluated and at least 500 cells were evaluated in each experiment. The percentage of BrdUpositive cells in the control vector (pBabe) was considered as 100 .
The error bars represent the standard deviation generated from three independent experiments.

\section{Cell cycle analysis}

Cells $\left(5 \times 10^{5}\right)$ were plated in 5\% FCS culture medium. After $24 \mathrm{~h}$, the culture medium was replaced by SFM for $48 \mathrm{~h}$. The cells were harvested by trypsinisation and then fixed in ice-cold $70 \%$ ethanol. The cells were then washed with PBS and incubated with propidium iodide $\left(50 \mu \mathrm{g} \mathrm{ml}^{-1}\right)$ and RNase $\left(1 \mu \mathrm{g} \mathrm{ml}^{-1}\right)$ for $30 \mathrm{~min}$. Flow cytometric analysis was performed on an EPICS profile analyzer and analysed using the ModFit LT2.0 software (Coulter) as described previously (Wang et al, 2002a).

\section{Western blotting}

Detailed experimental procedures were described previously (Ouyang et al, 2002b). Briefly, whole-cell lysate was prepared by resuspending cell pellet in lysis buffer $(50 \mathrm{~mm}$ Tris- $\mathrm{HCl}(\mathrm{pH} 8.0)$, $150 \mathrm{~mm} \mathrm{NaCl}, 1 \% \mathrm{NP} 40,0.5 \%$ deoxycholic acid, $0.1 \%$ SDS) including protease inhibitors $\left(1 \mu \mathrm{g} \mathrm{ml}^{-1}\right.$ aprotinin, $1 \mu \mathrm{g} \mathrm{ml}^{-1}$ leupeptin, $1 \mathrm{~mm}$ PMSF), and protein concentrations were measured using the protein assay kit (Bio-Rad, Hercules, CA, USA). Protein suspension from the whole-cell lysate $(20 \mu \mathrm{g})$ was loaded onto a sodium dodecylsulphate-polyacrylamide gel (SDS-PAGE) for electrophoresis and then transferred to a PVDF membrane (Amersham, Piscataway, NJ, USA). The membrane was then incubated with primary antibody for $1 \mathrm{~h}$ at room temperature against Id-1, EGFR, $\beta$-actin (Santa Cruz Biotechnology, Santa Cruz, CA, USA). After washing with TBS-T, the membrane was incubated with secondary antibody against mouse or rabbit IgG and the signals were visualised using ECL plus Western blotting system (Amersham, Piscataway, NJ, USA).

\section{Luciferase assay}

Cells were plated into a 12 -well plate at a density of $1 \times 10^{5}$ cells well ${ }^{-1}$. After $24 \mathrm{~h}$, the medium was changed to SFM. pER-1 (luciferase reporter containing the EGFR promoter, kindly provided by Dr A Johnson, NCI, MD, USA) and pRL-CMV-Luc (internal control) were cotransfected with either the pcDNA, pcDNA-Id-1 or pcDNA-Id-1-AS, respectively (Ling et al, 2004), into the cells using Fugene 6 reagent (Roche Diagnostics, Indianapolis, IN, USA). Cells were lysed $48 \mathrm{~h}$ after transfection and were assayed for luciferase activity using the Dual-luciferase reporter assay system (Promega, WI, USA). Each data point represented the mean of three experiments and error bars indicated the standard deviation.

\section{RESULTS}

Generation of stable Id-1 transfectants in ovarian cancer cells

Under in vitro culture conditions, Id-1 expression is usually dependent on FCS stimulation and this characteristic is more evident in cell lines exhibiting less aggressive phenotype. For example, the androgen- or oestrogen-dependent cell lines (i.e. LNCaP (prostate cancer) and MCF7 (breast cancer)), which represent less aggressive tumours, show serum-dependent Id-1 expression. In contrast, the androgen- or oestrogen-independent cell lines (i.e. PC3 (prostate cancer) and MDA-MB-231 (breast cancer)), which represent aggressive tumours, express the Id-1 protein constitutively regardless of serum concentrations (Lin et al, 2000; Ouyang et al, 2002b). This phenotype provides a tool for studying the direct role of Id-1 on human cancer cells through either ectopic introduction or inactivation of the Id-1 gene. In this study, we first examined Id-1 protein expression in five ovarian 


\section{A}

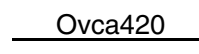

\begin{tabular}{r|llll} 
Serum (\%) & 5 & 2.5 & 1 & 0 \\
\hline Id-1 & & & \\
Actin & & &
\end{tabular}
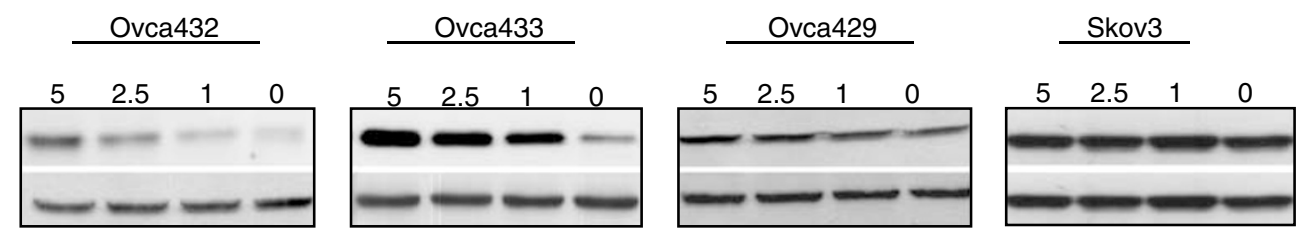

B
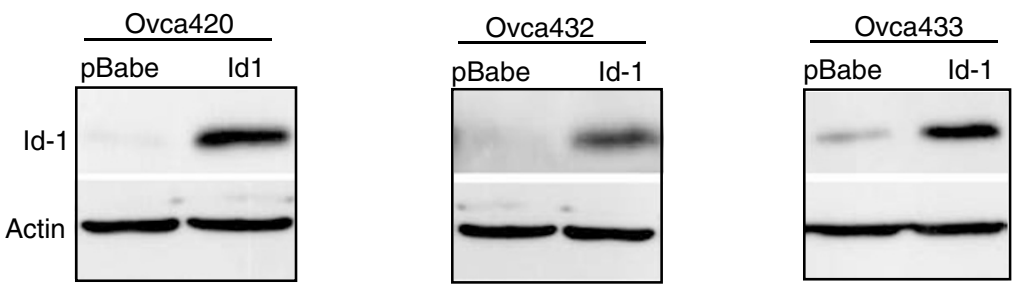

Figure I Effect of FCS on Id-I expression in ovarian cancer cell lines and Id-I expression in stable transfectants. (A) Western blotting analysis of Id- I protein expression in five ovarian cancer cell lines, Ovca420, Ovca432, Ovca433, Ovca429 and Skov3, in culture medium containing different serum concentrations. Note that the expression of Id-I is dependent on FCS in Ovca420, Ovca432 and Ovca433 cell lines but independent in Ovca429 and Skov3 cell lines. (B) Three cell lines Ovca420, Ovca432 and Ovca433 were transfected with an Id-I expression vector (pBabe-Id-I) or the empty control (pBabe) and stable transfectants were generated. Id- I protein expression was examined by Western blotting after culturing in SFM for $48 \mathrm{~h}$. Expression of actin was examined as an internal loading control. Note that Id-I protein levels are much higher after ectopic expression of the pBabe-ld-I vector in Ovca420, Ovca432 and Ovca433 cell lines. Results represent three experiments.

cancer cell lines in a range of FCS concentrations $(5,2.5,1$ and $0 \%$ ). As shown in Figure 1A, ovarian cancer cell lines Ovca420, Ovca432 and Ovca433 showed a serum-dependent Id-1 expression while the expression of Id-1 was much less serum dependent in the Ovca429 and Skov3 cells. The Id-1 expression pattern in these ovarian cancer cell lines also seemed to correlate with their malignant phenotype. For example, Ovca429 cell line has been reported to be much more aggressive demonstrated by its highly invasive nature and increased expression of metastasis promoting proteins such as uPA and MMP compared to Ovca432 and Ovca432 cells (Ellerbroek et al, 1998; Ahmed et at, 2002). We then transfected an Id-1 expression vector into Ovca420, Ovca432 and Ovca433 cell lines and generated stable transfectants. As shown in Figure 1B, after selection in puromycin, the Id-1 stable transfectants showed constitutively high levels of Id-1 protein expression compared to the vector controls when cultured in serum-free conditions.

\section{Effect of Id-1 expression on serum-independent cell proliferation in ovarian cancer cells}

To investigate the effect of Id-1 expression on ovarian cancer cell proliferation, the DNA synthesis rate and the cell cycle S-phase fraction were determined using BrdU staining and cell cycle analysis, respectively, in the cells cultured in SFM. As shown in Figure 2, after culturing in SFM for 48 and $72 \mathrm{~h}$, the percentage of BrdU-positive cells in Id-1 transfectants (solid columns) was higher than the vector controls (open columns) in all three ovarian cancer cell lines, especially in Ovca432 (up to 10-fold increase). The small increase of BrdU incorporation in Ovca433 Id-1 transfectant (approximately 1.7-2-fold) may be due to the relatively high basal levels of Id-1 in the parental cells, which may facilitate cell proliferation in SFM conditions. Nevertheless, these results indicate that ectopic expression of Id-1 in ovarian cancer cells led to increased DNA synthesis rate. To confirm these results, we performed flow cytometric analysis and studied the Sphase fraction. As shown in Figure 3, the Id-1 transfectants from all three cell lines showed higher percentage of S-phase cells after culturing in SFM for 48 and $72 \mathrm{~h}$, respectively. For example, the
A

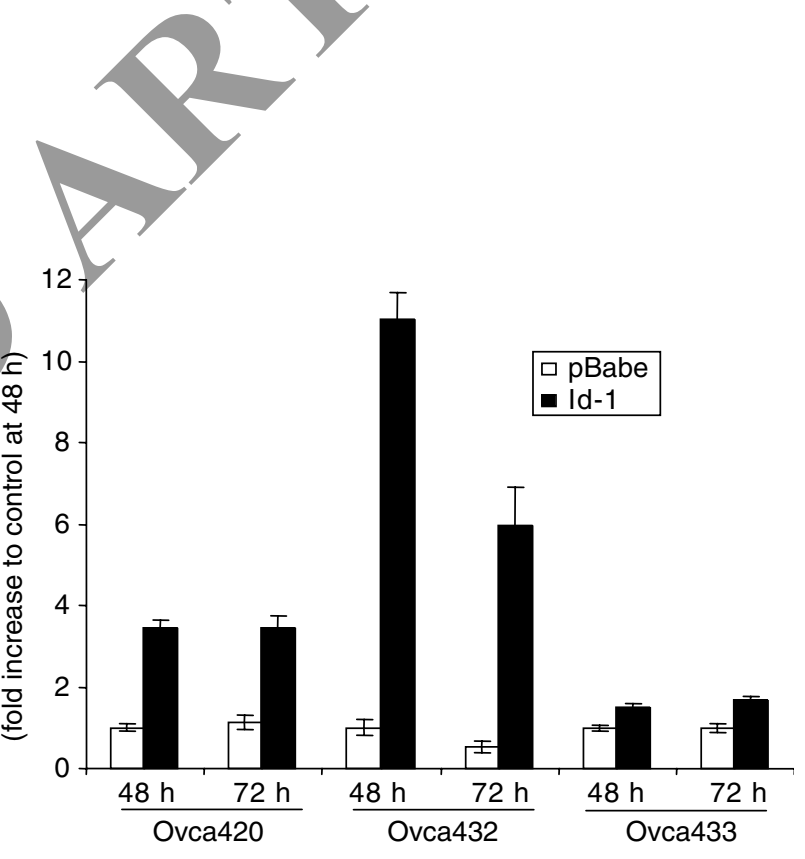

B

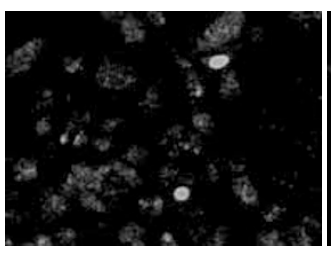

Ovca432 pBabe

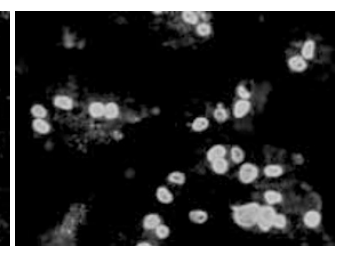

Ovca432 Id-1
Figure 2 Effect of ectopic Id-I expression on ovarian cancer cell proliferation. (A) BrdU incorporation rate between the Id-I transfectants and the vector controls. Cells were cultured in SFM for 48 and $72 \mathrm{~h}$, respectively, and stained with an antibody against BrdU. At least 500 cells were counted in each experiment and the percentage of BrdU-positive cells was calculated and compared with the vector controls at $48 \mathrm{~h}$ time point, which was assigned as I. Results presented as the mean and standard deviation from three experiments. (B) Representative results of $\mathrm{BrdU}$ staining. Strong nuclear FITC-positive signal was considered as BrdU positive. The background was enhanced to facilitate the counting of total cell numbers. Note that the percentage of BrdU-positive cells is higher in Id-I transfectants than the pBabe control transfectants. 


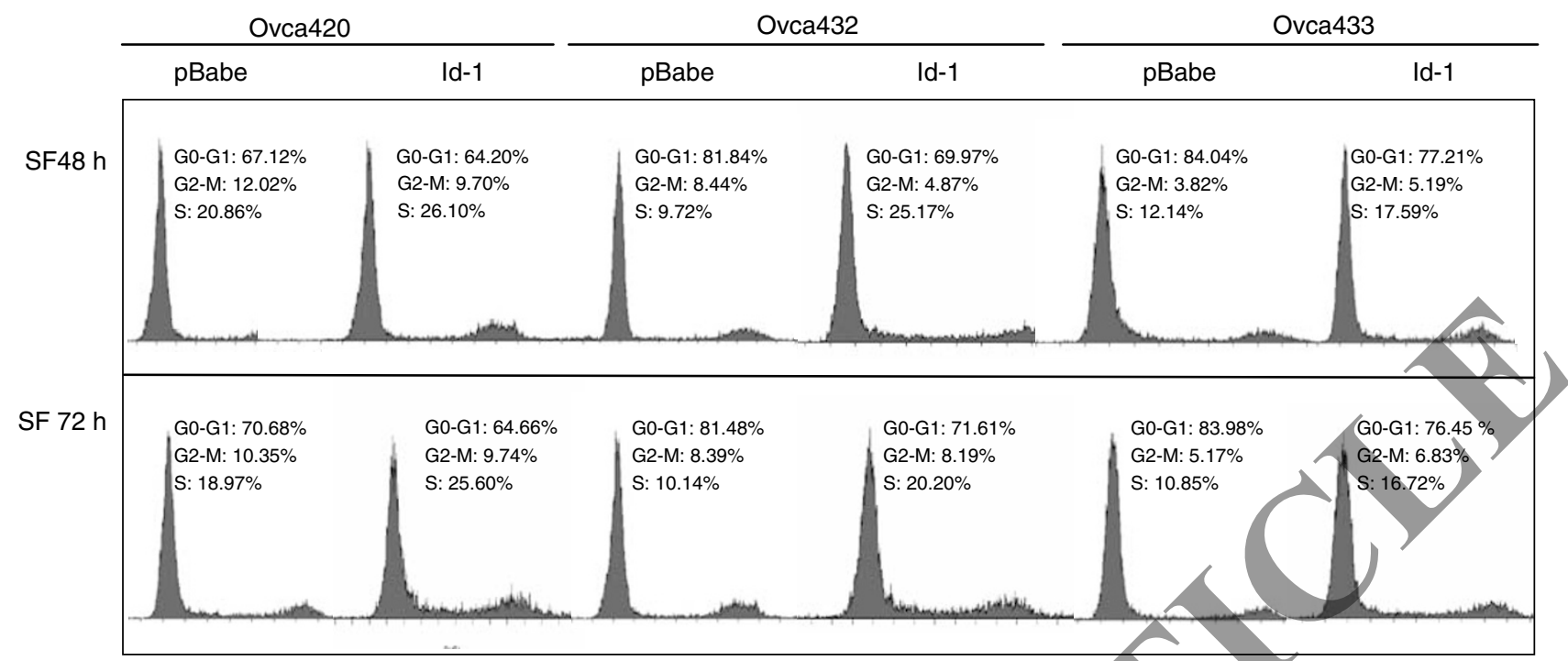

Figure 3 Effect of ectopic ld-I expression on cell cycle S-phase fraction. Flow cytometric analysis was performed on the Id-I and pBabe transfectants after culturing in SFM for 48 and $72 \mathrm{~h}$, respectively. Note that the percentage of S cells is higher in Id-I transfectants than in the vector control.

S-phase fraction was $25.17 \%$ in Ovca432-Id-1 transfectant, while the vector control only had $9.72 \%$ of S-phase cells after culturing in $\mathrm{SFM}$ for $48 \mathrm{~h}$. Taken together, these results suggest that upregulation of Id-1 promotes cell proliferation in ovarian cancer cells.

\section{Effect of ectopic Id-1 expression on EGFR in ovarian cancer} cells

As previously suggested, Id-1 promotes cell proliferation through EGFR pathway in prostate cancer cells (Ling et al, 2004), Next, we studied if the Id-1-induced cell proliferation in ovarian cancer cells was mediated through EGFR pathway. As shown in Figure $4 \mathrm{~A}$, the vector-transfected control Ovca420 and Ovca433 cells showed detectable basal levels of EGFR protein, while EGFR was absent in Ovca432 cells. After exogenous Id-1 expression, the EGFR protein level was increased remarkably in all of the Id-1 transfectants compared with the vector control, suggesting that ectopic Id-1 expression resulted in upregulation of EGFR protein expression.

To confirm these results, luciferase assay was performed to examine if ectopic Id-1 expression could lead to EGFR activation at transcriptional level. After cotransfection of pcDNA-Id-1 or pcDNA vectors with pER-1 (luciferase reporter containing the EGFR promoter) and pRL-CMV-Luc (internal control) in the parental cell lines, we found that the EGFR promoter activity was increased in all three cell lines transfected with the Id-1 expression vector (solid columns, 250-350\% increase) than the cells transfected with the pcDNA vector control (open columns) (Figure 4B). Taken together, these results indicate that upregulation of Id-1 in Ovca420, Ovca432 and Ovca433 cells has led to the upregulation of EGFR expression at both transcriptional and protein levels.

\section{Effect of antisense Id-1 on the expression of EGFR in ovarian cancer cells}

To further confirm the association between Id-1 and EGFR expression, we next studied whether blockage of Id-1 expression could lead to the downregulation of EGFR. As shown in Figure 1A, the Skov3 and Ovca429 cell lines had constitutively high levels of Id-1 expression regardless of serum concentrations; we then transfected a vector containing the antisense sequence to the Id-1 gene (Ling et al, 2003) into these two cell lines and tested the
A

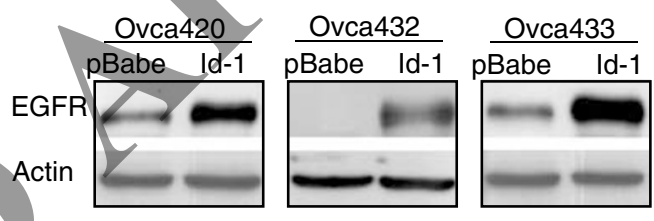

B

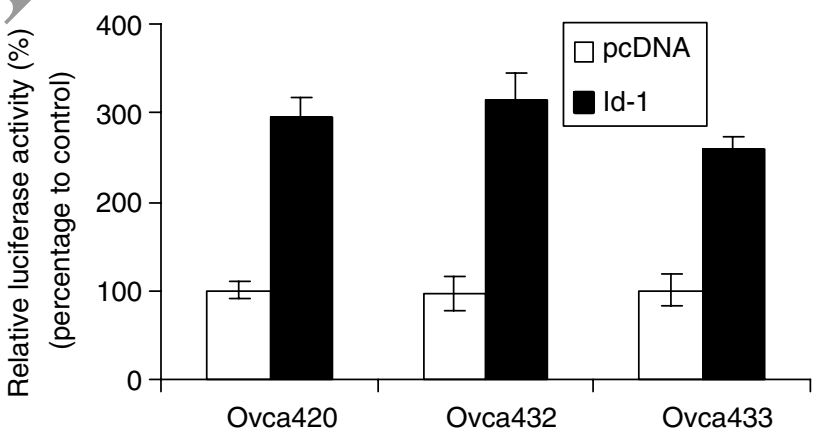

Figure 4 Effect of ectopic Id-I expression on EGFR. (A) EGFR protein expression in the Id-I transfectants and the vector control cells were cultured in SFM for $48 \mathrm{~h}$ and EGFR expression was examined by Western blotting. (B) EGFR promoter activity in Ovca420, Ovca432 and Ovca433 cells transiently transfected with pcDNAld-I and the vector control pcDNA. pER-I (luciferase reporter containing the EGFR promoter) and PRL-CMV-Luc (internal control) was cotransfected with PCDNA or pcDNA-ld-I, respectively. Cells were lysed $48 \mathrm{~h}$ after transfection and assayed for luciferase activity using the Dual-luciferase reporter assay system (Promega, WI, USA). The luciferase activity of the cells transfected with pER-I and pcDNA was considered as 100\%. Each experiment was repeated three times and the error bars represent the standard deviation from three independent experiments. Note that ectopic expression of Id- I in Ovca420, Ovca432 and Ovca433 cells induces upregulation of EGFR at both transcriptional and protein levels.

expression of EGFR. As shown in Figure 5A, $48 \mathrm{~h}$ after transfection, the Id-1 protein level was decreased in both cell lines compared to the pcDNA vector control (up to $70 \%$ decrease), indicating a successful inhibition of Id-1 expression in these cells. In addition, the expression of the EGFR protein was also downregulated at 
A
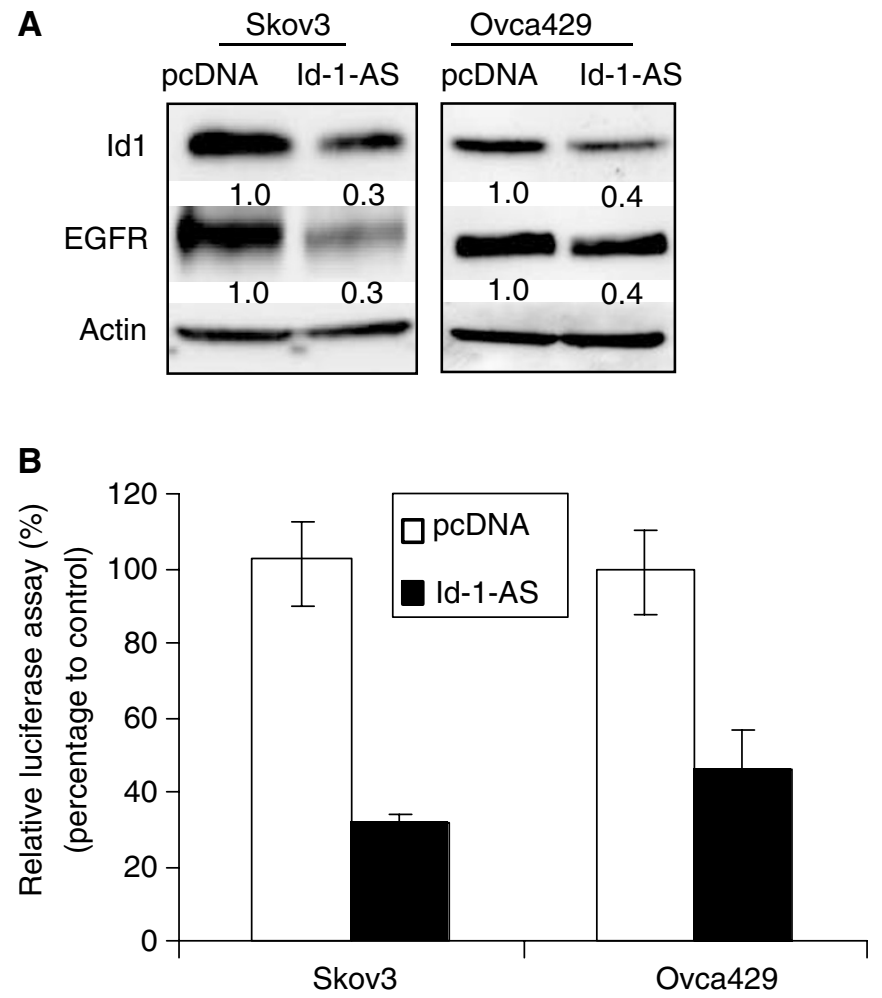

Figure 5 Effect of Id-I inactivation on EGFR. (A) Id-I and EGFR protein expression in ovarian cancer cells transiently transfected with an antisense Id-I expression vector (pcDNA-ld-I-AS) and the control vector (pcDNA). Cells were cultured in SFM for $48 \mathrm{~h}$ after transfection and Id-I and EGFR expression was examined by Western blotting. (B) EGFR promoter activity in ovarian cancer cells transiently transfected with IdI-AS and the vector control. pER-I (luciferase reporter containing the EGFR promoter) and pRL-CMV-Luc (internal control) were cotransfected with pcDNA or pcDNA-ld-I-AS, respectively, to Skov3 and Ovca429 cells. Cells were lysed for luciferase assays $48 \mathrm{~h}$ after transfection and the luciferase activity was tested using the Dual-luciferase reporter assay system (Promega, WI, USA). Samples transfected with pER-I and pcDNA was considered as 100\%. The error bars represent standard deviation from three independent experiments. Note that inhibition of Id-I expression in Skov3 and Ovca429 cells reduces EGFR expression at both transcriptional and protein levels.

similar levels in these two cell lines. For example, Id-1 expression was decreased by $70 \%$ in Skov 3 cells and the EGFR protein level was also reduced by approximately $70 \%$ compared to the vectortransfected cells. These results were further confirmed by luciferase assay, which showed that the EGFR promoter activity was inhibited in the cells transfected with the antisense Id-1 vector (solid columns) compared to the vector control (open columns) (Figure 5B). These results further support the hypothesis that Id-1 may be an upstream regulator of the EGFR signalling pathway.

\section{DISCUSSION}

In this study, we have demonstrated the positive effect of Id-1 expression on cell proliferation in three ovarian cancer cell lines (Figures 1-3). In addition, the evidence provided in this study that ectopic expression (or downregulation) of Id-1 led to upregulation (or downregulation) of EGFR at both transcriptional and protein levels indicates that Id-1 may be an upstream positive regulator of the EGFR signalling pathway (Figures 4 and 5). Since upregulation of EGFR is a common event in ovarian cancer, our evidence implicates a novel mechanism responsible for EGFR activation in this cancer. As EGFR is one of the key factors in promoting ovarian cancer cell growth (Alper et al, 2000, 2001; Pack et al, 2004), our results also suggest an alternative target to suppress EGFR signalling pathway through inactivation of Id-1, thus inhibiting cancer cell growth. Although the Id-1-induced cell proliferation in normal as well as in cancer cells has been reported in several previous studies (Lin et al, 2000; Ouyang et al, 2002b; Wang et al, $2002 b)$, this study is the first to demonstrate its positive role on ovarian cancer cell growth, especially its relation with EGFR pathway in ovarian cancer cells.

Several mechanisms have been suggested for the role of Id-1 in cell proliferation. For example, Id-1 inhibits p16 $6^{\text {INK4a }} / \mathrm{RB}$ pathway leading to the bypass of replicative senescence in primary cells (Alani et al, 2001; Ohtani et al, 2001) and induces serumindependent proliferation in human prostate cancer cells (Ouyang et al, 2002b). Recently, the Id-1-induced cell proliferation has been linked to the Raf-MEK and NF- $\kappa \mathrm{B}$ pathways (Ohtani et al, 2001; Ling et al, 2002, 2003). Since Id-1 is a regulator of transcription, it is not surprising that it may regulate gene expression involving multiple signalling pathways. In this study, we found that ectopic Id-1 expression led to transcriptional activation of EGFR (Figure 4), while inactivation of Id-1 resulted in suppression of EGFR expression (Figure 5). Our results indicate a novel downstream effector of Id-1 in ovarian cancer cells. It is possible that increased Id-1 expression in ovarian cancer cells may provide autocrine signals to stimulate EGFR activity, resulting in promotion of cell proliferation. Since inactivation of EGFR has shown promising results in inhibition of ovarian cancer cell growth as well as suppression of metastatic phenotypes, our results suggest a new target in inhibition of EGFR signalling. Previously, it was also reported that Id-1 protected against anticancer drug taxol-induced cell death in nasopharyngeal carcinoma cells (Cheung et al, 2004) and suppression of Id-1 resulted in sensitisation to TNF $\alpha$-induced apoptosis in prostate cancer cells (Ling et al, 2003). Since the frontline treatment strategy for advanced ovarian cancers is chemotherapy, downregulation of Id-1 may provide a novel strategy in improving the efficiency of chemotherapeutic drugs through suppression of Id-1-induced protection against apoptosis.

Activation of EGFR as well as upregulation of Id-1 have been associated with aggressive behaviour and poor clinical outcome in ovarian cancer patients, respectively (Schindl et al, 2003; Skirnisdottir et al, 2004). In this study, we also found that the cell lines (i.e. Ovca432, Ovca433) that showed serum-dependent Id1 expression had lower basal levels of EGFR and exhibited less invasive characteristics as reported in previous studies than the ones with serum-independent Id-1 expression (i.e. Ovca429) (Ellerbroek et al, 1998; Ahmed et al, 2002). These results further suggest a strong link between Id-1 and EGFR in promoting ovarian cancer progression. Recently, the positive role of Id-1 in metastasis has been reported through promoting tumour angiogenesis in a breast cancer animal model (Fong et al, 2003). In ovarian cancer, similar effects have been reported in the cells expressing high levels of EGFR. For example, Ovca8 cells expressing high levels of EGFR were much more invasive and contained high levels of integrins and MMP activity and these malignant phenotypes were suppressed when EGFR was inactivated through antisense technology (Alper et al, 2001). It is possible that increased expression of Id-1 and EGFR proteins may provide growth advantage for ovarian cancer cells to progress to more advanced malignant phenotype leading to poor prognosis in patients. However, how Id-1 and EGFR interact with each other and what signalling pathways are responsible for mediating ovarian cancer proliferation and progression remain to be elucidated.

In summary, we have provided first evidence that Id-1 plays an important part in the proliferation of ovarian cancer cells and this function is mediated through upregulation of EGFR. Although further investigations are needed to elucidate the precise molecular mechanisms responsible for the role of Id-1 in ovarian cancer, our 
results suggest a novel upstream regulator of the EGFR pathway. Since inhibition of EGFR is effective in the suppression of ovarian cancer cell growth, inactivation of Id-1 may provide an alternative strategy for the treatment of this cancer.

\section{ACKNOWLEDGEMENTS}

This work was supported by RGC Grants to XH Wang (HKU7478/ 03M) and YC Wong (HKU 7314/01M and HKU7490/03M).

\section{REFERENCES}

Alani RM, Young AZ, Shifflett CB (2001) Id1 regulation of cellular senescence through transcriptional repression of p16/Ink4a. Proc Natl Acad Sci USA 98: 7812-7816

Alper O, Bergmann-Leitner ES, Bennett TA, Hacker NF, Stromberg K, Stetler-Stevenson WG (2001) Epidermal growth factor receptor signaling and the invasive phenotype of ovarian carcinoma cells. J Natl Cancer Inst 93: $1375-1384$

Alper O, De Santis ML, Stromberg K, Hacker NF, Cho-Chung YS, Salomon DS (2000) Anti-sense suppression of epidermal growth factor receptor expression alters cellular proliferation, cell-adhesion and tumourigenicity in ovarian cancer cells. Int J Cancer 88: 566-574

Ahmed N, Pansino F, Baker M, Rice G, Quinn M. (2002) Association between alphavbeta6 integrin expression, elevated p42/44 kDa MAPK, and plasminogen-dependent matrix degradation in ovarian cancer. $J$ Cell Biochem 84: $675-686$

Benezra R, Davis RL, Lockshon D, Turner DL, Weintraub H (1990) The protein Id: a negative regulator of helix-loop-helix DNA binding proteins. Cell 61: $49-59$

Cheung HW, Ling MT, Tsao SW, Wong YC, Wang X (2004) Id-1-induced Raf/MEK pathway activation is essential for its protective role against taxol-induced apoptosis in nasopharyngeal carcinoma cells. Carcinogenesis 25: $881-887$

Cloven NG, Kyshtoobayeva A, Burger RA, Yu IR, Fruehauf JP (2004) In vitro chemoresistance and biomarker profiles are unique for histologic subtypes of epithelial ovarian cancer. Gynecol Oncol 92: 160-166

Ellerbroek SM, Hudson LG, Stack MS (1998) Proteinase requirements of epidermal growth factor-induced ovarian cancer cell invasion. Int $\mathrm{J}$ Cancer 78: $331-337$

Fong S, Itahana Y, Sumida T, Singh J, Coppe JP, Liu Y, Richards PC, Bennington JL, Lee NM, Debs RJ, Desprez PY (2003) Id-1 as a molecular target in therapy for breast cancer cell invasion and metastasis. Proc Natl Acad Sci USA 100: $13543-13548$

Jemal A, Tiwari RC, Murray T, Ghafoor A, Samuels A, Ward E, Feuer EJ, Thun MJ, American Cancer Society (2004) Cancer statistics. CA Cancer J Clin 54: 8-29

Kohler M, Janz I, Wintzer HO, Wagner E, Bauknecht T (1989) The expression of EGF receptors, EGF-like factors and c-myc in ovarian and cervical carcinomas and their potential clinical significance. Anticancer Res 9: $1537-1547$

Langlands K, Down GA, Kealey T (2000) Id proteins are dynamically expressed in normal epidermis and dysregulated in squamous cell carcinoma. Cancer Res 60: 5929-5933

Lin CQ, Singh J, Murata K, Itahana Y, Parrinello S, Liang SH, Gillett CE, Campisi J, Desprez PY (2000) A role for Id-1 in the aggressive phenotype and steroid hormone response of human breast cancer cells. Cancer Res 60: $1332-1340$

Ling MT, Wang X, Lee DT, Tam PC, Tsao SW, Wong YC (2004) Id-1 expression induces androgen independent prostate cancer cell growth through activation of epidermal growth factor receptor (EGF-R). Carcinogenesis 25: $517-525$

Ling MT, Wang X, Ouyang X, Lee TKW, Fan T, Xu K, Tsao SW, Wong YC (2002) Activation of MAPK signaling pathway is essential for Id-1 induced serum independent prostate cancer cell growth. Oncogene 21: $8498-8505$

Ling MT, Wang X, Ouyang XS, Xu K, Tsao SW, Wong YC (2003) Id-1 expression promotes cell survival through activation of NF-kB signalling pathway in prostate cancer cells. Oncogene 22: 4498-4508
Maihle NJ, Baron AT, Barrette BA, Boardman CH, Christensen TA, Cora EM, Faupel-Badger JM, Greenwood T, Juneja SC, Lafky JM, Lee H, Reiter JL, Podratz KC (2002) EGF/ErbB receptor family in ovarian cancer. Cancer Treat Res 107: 247-258

Maruyama H, Kleeff J, Wildi S, Friess H, Buchler MW, Israel MA, Korc M (1999) Id-1 and Id-2 are overexpressed in pancreatic cancer and in dysplastic lesions in chronic pancreatitis. Am J Pathol 155: $815-822$

Ohtani N, Zebedee Z, Huot TJ, Stinson JA, Sugimoto M, Ohashi Y, Sharrocks AD, Peters G, Hara E (2001) Opposing effects of Ets and Id proteins on p16INK4a expression during cellular senescence. Nature 409: $1067-1070$

Ouyang XS, Wang X, Lee DT, Tsao SW, Wong YC (2002a) Over expression of ID-1 in prostate cancer. J Urol 167: 2598-2602

Ouyang XS, Wang X, Ling MT, Wong HL, Tsao SW, Wong YC (2002b) Id-1 stimulates serum independent prostate cancer cell proliferation through inactivation of p16(INK4a)/pRB pathway. Carcinogenesis 23: $721-725$

Pack SD, Alper OM, Stromberg K, Augustus M, Ozdemirli M, Miermont AM, Klus G, Rusin M, Slack R, Hacker NF, Ried T, Szallasi Z, Alper O (2004) Simultaneous suppression of epidermal growth factor receptor and c-erbB-2 reverses aneuploidy and malignant phenotype of a human ovarian carcinoma cell line. Cancer Res 64: 789-794

Parkin DM, Bray F, Ferlay J, Pisani P (2001) Estimating the world cancer burden: Globocan 2000. Int J Cancer 94: 153-156

Rauh-Adelmann C, Lau KM, Sabeti N, Long JP, Mok SC, Ho SM (2000) Altered expression of BRCA1, BRCA2, and a newly identified BRCA2 exon 12 deletion variant in malignant human ovarian, prostate, and breast cancer cell lines. Mol Carcinogen 28: 236-246

Schindl M, Oberhuber G, Obermair A, Schoppmann SF, Karner B, Birner P (2001) Overexpression of Id-1 protein is a marker for unfavorable prognosis in early-stage cervical cancer. Cancer Res 61: 5703-5706

Schindl M, Schoppmann SF, Strobel T, Heinzl H, Leisser C, Horvat R, Birner P (2003) Level of Id-1 protein expression correlates with poor differentiation, enhanced malignant potential, and more aggressive clinical behavior of epithelial ovarian tumours. Clin Cancer Res 9: $779-785$

Sewell JM, Macleod KG, Ritchie A, Smyth JF, Langdon SP (2002) Targeting the EGF receptor in ovarian cancer with the tyrosine kinase inhibitor $\mathrm{ZD}$ 1839 ('Iressa'). $\mathrm{Br}$ J Cancer 86: 456-462

Simpson BJ, Phillips HA, Lessells AM, Langdon SP, Miller WR (1995) c-erbB growth-factor-receptor proteins in ovarian tumours. Int J Cancer 64: $202-206$

Skirnisdottir I, Seidal T, Sorbe B (2004) A new prognostic model comprising p53, EGFR, and tumour grade in early stage epithelial ovarian carcinoma and avoiding the problem of inaccurate surgical staging. Int J Gynecol Cancer 14: 259-270

Skirnisdottir I, Sorbe B, Seidal T (2001) The growth factor receptors HER-2/ neu and EGFR, their relationship, and their effects on the prognosis in early stage (FIGO I-II) epithelial ovarian carcinoma. Int J Gynecol Cancer 11: $119-129$

Wang X, Jin DY, Ng RW, Feng H, Wong YC, Cheung AL, Tsao SW (2002a) Significance of MAD2 expression to mitotic checkpoint control in ovarian cancer cells. Cancer Res 62: $1662-1668$

Wang X, Xu K, Ling MT, Wong YC, Feng HC, Nicholls J, Tsao SW (2002b) Evidence of increased Id-1 expression and its role in cell proliferation in nasopharyngeal carcinoma cells. Mol Carcinogen 35: $42-49$ 